\title{
Attenuated Total Reflectance-Fourier-Transform Infrared Microspectroscopy a Rapid Method for Microbial Strain Characterization
}

\author{
Meshref Awad Alruwaili \\ College of Applied Medical Science, Aljouf University, Clinical Lab. Science, B.O. 2014, Skaka, KSA
}

Received 2013-05-01, Revised 2013-05-21; Accepted 2013-06-08

\begin{abstract}
Fourier-Transform Infrared (FTIR) spectroscopy and BHI 50CH were used to identify bacteria of the Lactobacillus (L.) at the species level. A previously developed method for measuring FTIR spectra and a strategy for their analysis provided the basis for selecting the FTIR spectra of reference bacterial strains and created a spectral library. The research was launched in which the spectra collected in the above library were used for developing a spectral reference for known reference bacterial strains and the practical value of the generated library was verified based on the results of identification of four bacterial strains viz. L. plantarum, L. casei, L. lactis and L. fermentum of known taxonomy as well as identification of 15 bacterial strains isolated from rumen extracts and identified on the basis of their taxonomy and biochemical tests. The application of prepared lactic acid bacteria reference library for analysis of advanced analysis of FTIR was provided an accurate identification of $90 \%$ of bacterial strains of the genus Lactic acid bacteria identified by FTIR-microspectroscopy.
\end{abstract}

Keywords: Lactic Acid Bacteria, FTIR Spectra, Rumen Extract, Bacterial Isolation

\section{INTRODUCTION}

The latest methods for microorganism differentiation and identification are oriented towards simplicity, reducing the time needed to perform analysis towards high specificity and quality of microbial identification results. One of the methods that meet these criteria is Fourier-Transform Infrared (FTIR) spectroscopy. Studies conducted by (Dziuba et al., 2006; 2007; Mossoba et al., 2003; Nauman, 2000; Chohan et al., 2010) showed that FTIR spectra can be treated as specific to particular bacterial strains. They reflect the distinguishing features of all bacterial cell components, such as fatty acids, membrane and intracellular proteins, polysaccharides and nucleic acids. The differences between FTIR spectra of microorganisms are difficult to observe, thus referring to creative reference library and proper statistical methods are required for accurate and acceptable identification of isolated microorganisms (Dziuba, 2007; Samelis et al., 2010).
However, results obtained by FTIR are acceptable and dependent on the interpretation of spectrum through similarities with spectrum of known strains (Johnsen and Nielsen, 1999; Timmins et al., 1998; Huleihel et al., 2009). The results of the identification of bacterial spectra obtained by the above methods are comparable to those obtained with the use of other techniques such as biochemical identification method (API 50 CHL). Apart from classical methods, FTIR method is often applied for microorganism identification (Kirschner et al., 2001; Udelhoven et al., 2000; Dziuba et al., 2006; 2007; Sameli et al., 2011). Among numerous reports on microorganism differentiation, classification and identification using FTIR spectroscopy (Weinrichter et al., 2001; Igisu et al., 2009) a few only deal with lactic acid bacteria. However, the FTIR spectral characteristics of lactic acid bacteria was based exclusively on spectral differentiation index to describe the relationships between FTIR spectra and bacteria as molecular systems in a way that would permit their proper microbial 
identification. Generally FTIR/FTIR-ATR acquired spectra of bacteria are composed of hundreds or even thousands of overlapping bands that cannot be separated. It follows that their analysis requires the application of pattern recognition techniques, analyzing spectra as fingerprints. One of the most advanced and promising methods of this group is to create a reference library with organisms of concern, therefore, the objective of this study was to use FTIR/FTIR-ATR to create proper reference library and to differentiate microbial strains with particular attention to lactic acid bacteria as strains with Probiotic features used for several health attributes.

\section{MATERIALS AND METHODS}

\subsection{Bacterial Strains and Growth Conditions}

L. plantarum (ATTC 8014), L. casei (ATTC 303), L.actobacillus lactis (ATTC 7830) and L. fermentum (ATTC 9338) strains used in the study were purchased from international collections ATCC (Microbiologics, Medimark, Europe, France), microbial strain collection from the Department of Food Science, University of Manitoba, Canada (UoM) and strains isolated from rumen extracts of sheep, goat, camel and cattle that collected from different slaughterhouses located in northern and southern part of Jordan. API 50CHL and API 50 CHL medium were purchased from Biomerieux (BioMèrieux, Durham, USA).Bacterial strains were cultured anaerobically in solid MRS agar media for $48 \pm 2 \mathrm{~h}$ under optimum conditions at $30^{\circ} \mathrm{C}$.

\subsection{Sample Preparation, Measurement and Evaluation}

Reference and isolated microorganism cultures were grown on agar plate for $24 \mathrm{~h}$ and the cell material is harvested from the agar plate and suspended in sterilized physiological saline. A $120-\mu \mathrm{L}$ of the bacterial suspension was loaded onto special reusable micro plates in 96 well formats and dried for $30 \mathrm{~min}$ at $50^{\circ} \mathrm{C}$. FTIR measurements were performed in transmission mode. All spectra were recorded between 4000 and $600 \mathrm{~cm}^{-1}$ with a Tensor 27 FTIR spectrometer, equipped with HTS-XT accessory for rapid automation of the analysis (Bruker Optics, GmbH, Germany). Spectral resolution was set at $4 \mathrm{~cm}^{-1}$, sampling 256 scans per sample. After drying, the plate was inserted into Bruker Optics' microplate reader HTS-XT and scanned between 500 to $4000 \mathrm{~cm}^{-1}$. The measurements were repeated for four replicates. The acquired spectrum were evaluated using OPUS 5.5 software and compared with previously created spectrum similarities of different isolated microorganism for lactic acid bacteria of known strains and with those of ATTC.

Three samples of two independent cultures (a total of six samples) were prepared for each strain. The experiment was conducted in three replications. A biochemical tests using API 50CHL were also performed to compare FTIR with biochemical bacterial identification.

\subsection{FTIR/ATR-FTIR for Microbial Identification}

Microbial identification of food product is essential to verify the safety of commercially distributed food commodities for human consumption as well as to determine how effective of food processing in killing food spoilage or food-borne pathogens. ATR/FTIR micro spectroscopy capable of identifying microorganism through infrared spectrum of the biomolecules found specific for every microbial strain found within bacterial cells with fast, reliable, cheap compared with known other molecular or biochemical with customizable generated libraries. Attenuated Total Reflectance (ATR) Fourier transform infrared spectra were collected with a bench-top spectrometer (Tensor 27, Bruker Optics GmbH, Ettlingen, Germany). Spectra were recorded in a range from $850-4000 \mathrm{~cm}^{-1}$ at a resolution of $4 \mathrm{~cm}^{-1}$ and with an aperture of $6 \mathrm{~mm}$. Data storage, spectra processing, substance comparison and the quantitative analysis of the spectra were done with the software OPUS 6.5 (Bruker Optics, GmbH, Ettlingen, Germany).

\subsection{Database Library Creation}

The quality of the databases has a fundamental importance for the reliability of the identification. After all, each spectrum of an unknown microorganism is compared against all spectra from a created library for the known strains. A database library has to contain all relevant species, as well as all spectral variances of different strains from a single species. Food relevant microorganisms from reference stocks are not the only strains in the library, but the data of isolates from different production sites are also included. Prior to building up libraries, all microorganisms in the database were identified through reliable reference techniques.

\subsection{Data Analysis}

The recorded FTIR spectra together with the results from API $50 \mathrm{CHL}$ reference analysis were analyzed using partial Least-Squares (LSD) regression using SAS version 9. Spectrum during preparation of the calibration 
models were visually checked for principal component (Weinrichter et al., 2001). The spectral data were divided into three sets (learning, validation and testing) to include the spectra of all strains analysed. The validation set consisted of FTIR spectra of 4 reference strains. The testing set included 4 spectra of reference strains of known taxonomy and 7 spectra of bacteria isolated from the rumen extract. For the identification of Lactobacillus strains at the species level, the output layer consisted of ten neurons with assigned classes. The bacterial spectra were stored in a generated library for L. casei, L. delbrueckii. Subspecies lactis; $L$. fermentum and L. plantarum. Neurons organized in the layers, were connected in a way specific to a given type of artificial neural network.

\section{RESULTS}

The spectral differentiation of bacteria of the genus lactic acid bacteria of the isolated or ATCC collections strains are presented in Fig. 1 and 2 It is obvious that reference bacterial spectrum used for the identification of lactic acid bacteria in the present study were useful and gives and accurate results. One of the advantages of Bacterial Reference Spectrum (BRS) is their capability to generate knowledge to new strains with previously unseen bacterial strains, not fed into the reference library. At the same time BRS are able to memorize the acquired knowledge, which can be used at any moment without the need to feed the information again.

\section{DISCUSSION}

The process of bacterial identification involved bacterial strains assigned classes had been divided into three sets of validation and testing spectral techniques. The spectra from the validation served to control the identification process and to evaluate the validity of differentiation process. The capability to generalize the knowledge acquired during the tests was tested on the basis of the spectra contained in the testing. A total of 100 spectra were used during the development of artificial reference library for lactic acid bacterial identification at the species level. The acquired set was composed of 100 FTIR spectra, while the validation lactic acid bacterial groups were 10 FTIR spectra of 4 reference strains. The testing set included 20 spectra of reference strains, 4 spectra of bacteria of known taxonomy and 8 spectra of bacteria isolated from collected animal rumen extracts. The numbers of spectra of each lactic acid bacterial strains of reference strains and from bacterial species isolated from the rumen extracts were similar to be in balance in their demonstration along with sensitivity analysis to predict the proper spectral strains with specific variables based on the polysaccharide spectrum. The greatest extent of identifying bacterial strains with FTIR compared with biochemical test kit (API CHL 50). The selected variables provided the basis for creating another neural network. The trained network enabled to obtain fully correct results. The correctness of identification of Lactobacillus strains at the species level was verified according to a two-stage procedure. At the first stage artificial neural networks were tested based on the spectra of 4 reference strains. At the second stage the spectra of 4 strains of known taxonomy and 10 isolated strains, identified with biochemical tests (APICHL 50), were used. All reference spectral libraries were generated on FTIR spectra of the tested lactic acid bacteria. Similarly as in the case of bacteria identification at the genus level (Dziuba et al., 2007; Naumann et al., 1991; Sameli et al., 2011), the optimum reference bacterial library were selected as a consequence of searching for the most relevant lactic acid bacterial parameters and spectral reference similar to the isolated bacteria. The RBS presented in Fig. 1 and $\mathbf{2}$ correctly identified all reference strains of testing set $\mathrm{I}$, whereas bacteria of testing set II were correctly identified in 89 to $93 \%$ of cases. The best results were achieved using reference bacterial spectra for a combination of the spectral ranges. The reference bacterial correctly identified all reference strains and all strains of known taxonomy. The affiliation of the following four strains was not determined unambiguously: Lb. casei, Lb. fermentum, Lb. plantarum and $\mathrm{Lb}$. delbrueckii subspecies lactis, respectively. It was found that differentiation of Lactobacillus strains at the species level based on analysis of individual spectral regions and the entire spectrum was limited. The present results confirmed the correctness of conclusions drawn from a comparison of bacterial spectra determined on the basis of the differentiation index, but also proved the existence of such combinations of spectral ranges which permitted the most reliable identification of bacterial strains. The best results of differentiation of bacteria of the genus Lactobacillus at the species level were obtained for combinations of the ranges of the first derivatives of spectra for the polysaccharide region, the fingerprint region and the mixed region. Curk et al. (1994); Weinrichter et al. (2001) and Sameli et al. (2011) also achieved the best results for the above combinations. 

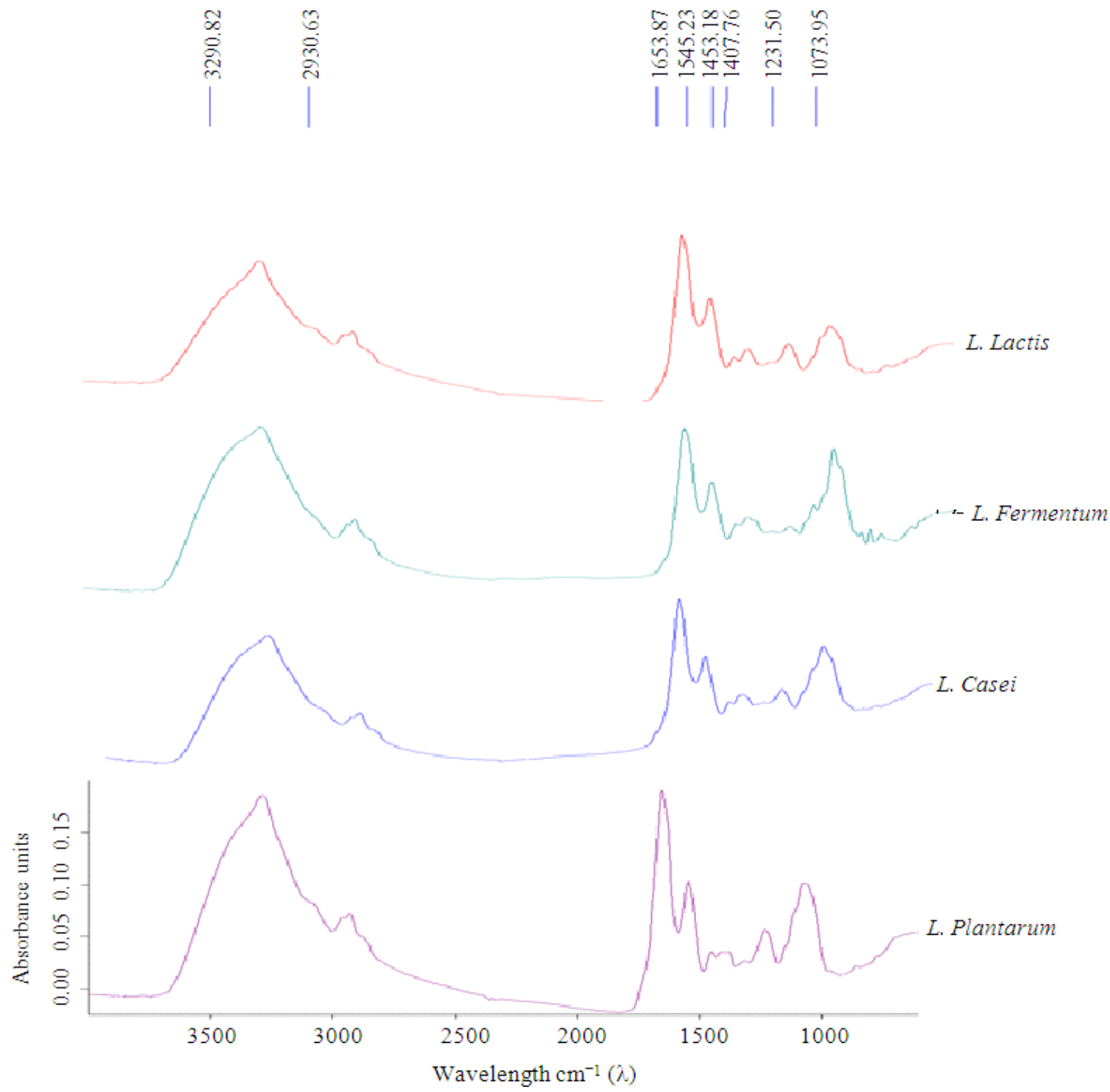

Fig. 1. FTIR absorbance spectra in the region from 1,800 to $900 \mathrm{~cm}^{-1}$ for intact Reference lactic acid bacteria gram-positive rods grown on MRS agar at $37^{\circ} \mathrm{C}$ for $24 \mathrm{~h}$. Pure ATTC strains from $L$ casei and L. plantarum. The bands indicated with arrows correspond to the main absorption bands assigned of the lactic acid bacteria.

However, these authors did not use artificial neural networks and we demonstrated previously (Dziuba, 2007; Mariey et al., 2001; Rodriguez-Saona et al., 2001) that the FTIR spectral characteristics of bacteria based exclusively on the differentiation index $\mathrm{D}$ and cluster analysis are not sufficient to describe the relationships between FTIR spectra and bacteria as molecular systems in a way that would permit their proper identification.
The application ATR-FTIR of a reference library and created reference strains spectrum enabled correct identification of $93 \%$ of bacterial strains of the used Lactic acid bacteria. Moreover, the results could be probably improved if the number of strains was increased, preferably including the so-called typical strains. A promising solution is to develop multilevel artificial neural networks, forming a single structure. The networks organized in this way would enable identifying microorganisms at various taxonomic levels. 


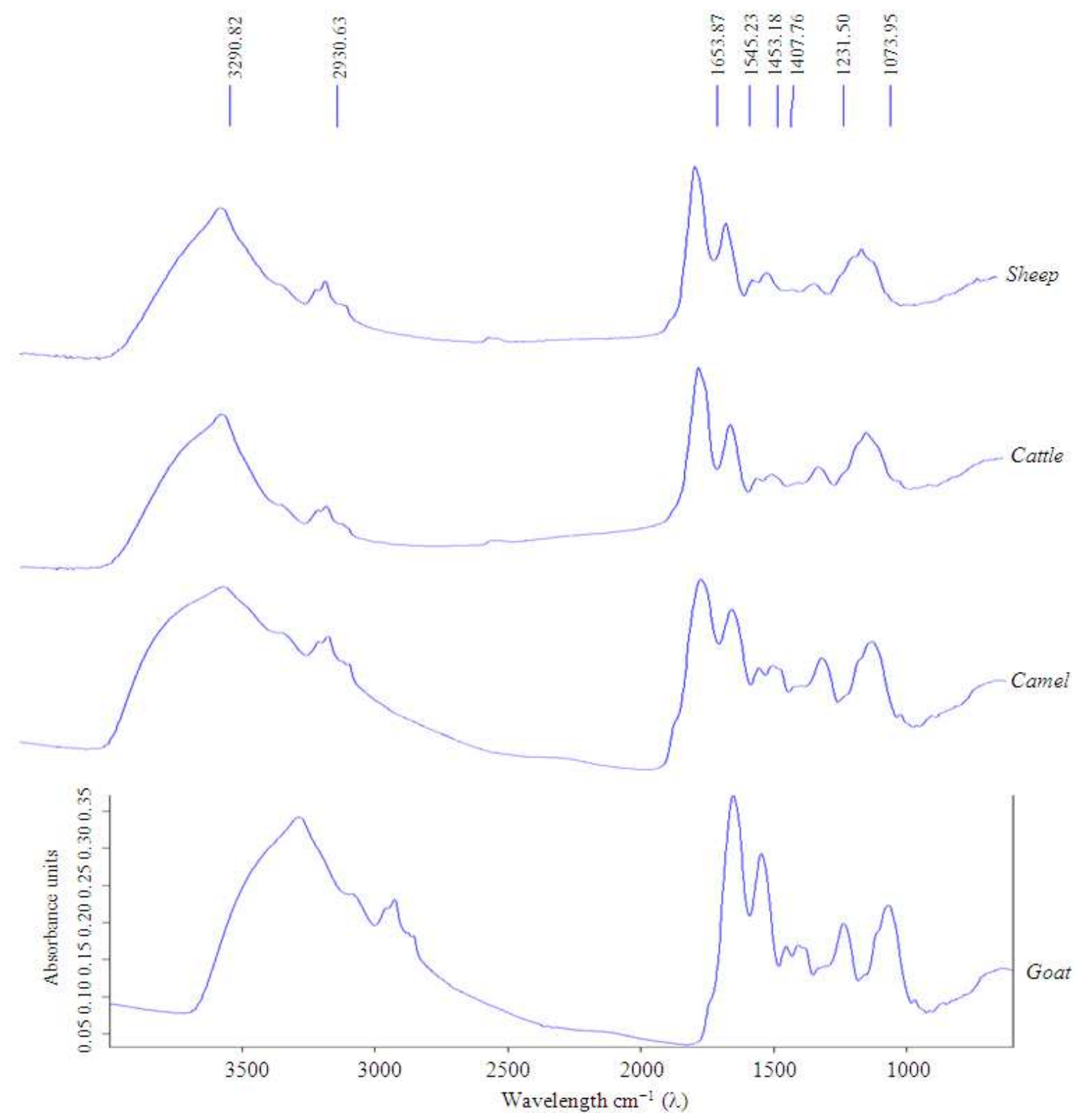

Fig. 2. FTIR absorbance spectra in the region from 1,800 to $900 \mathrm{~cm} \_1$ for intact Reference lactic acid bacteria gram-positive rods grown on MRS agar at $37^{\circ} \mathrm{C}$ for $24 \mathrm{~h}$. (a) Isolated strains from rumen extract of Sheep, Cattle Camel and Goat. The bands

\section{CONCLUSION}

- The measurement region of FTIR at wavelength between $1200 \mathrm{~cm}^{-1}$ to $900 \mathrm{~cm}^{-1}$ for bacterial strains polysaccharide with the specific region for differentiation between $900 \mathrm{~cm}^{-1}-700 \mathrm{~cm}^{-1}$ and regions $1500 \mathrm{~cm}^{-1}$ to $1200 \mathrm{~cm}^{-1}$ of the bacterial acquired were found most appropriate spectra for FTIR characterizing strains of lactic acid bacteria

- The application FTIR reference bacterial strains spectrum library is considered as method of choice in using FTIR for correct identification of lactic acid bacterial strains with $>90$ confidence limit 
- ATR-FTIR/FTIR spectroscopy, combined with reference library was found to be suitable, simple, fast and accurate technique in microbial identification of lactic acid and could be used in identification of food borne pathogens and food spoilage bacteria at strain level and seems to be very promising

\section{ACKNOWLEDGEMENT}

The researchers gives special thanks to Prof. Dr. Holley from The University of Manitoba for providing support, space and opportunity to work and write the manuscript and to the Deanship of Academic Research at The University of Jordan for providing their support and bacterial strains for this study. Thanks also to Mu'tah and Al-jouf Universities for providing their facilities to complete the research.

\section{REFERENCES}

Chohan, Z. H., M. H. Youssoufi, A. J. and T. B. Hadda, 2010. Identification of antibacterial and antifungal pharmacophore sites for potent bacteria and fungi inhibition: Indolenyl sulfonamide derivatives. Eur. J. Med. Chem., 45: 1189-1199. DOI: 10.1016/j.ejmech.2009.11.029

Curk, M.C., F. Peladan and J.C. Hubert, 1994. Fourier Transform Infrared (FTIR) spectroscopy for identifying Lactobacillus species. FEMS Microbiol. Lett., 123: 241-248. DOI: 10.1111/j.15746968.1994.tb07231.x

Dziuba, B., 2007. Identification of Lactobacillus strains at the species level using FTIR spectroscopy and artificial neural networks. Pol. J. Food Nutr. Sci., 57: 301-306.

Dziuba, B., A. Babuchowski, M. Niklewicz and B. Brzozowski, 2006. FTIR spectral characteristics of lactic acid bacteria: A spectral library. Milchwissenschaft, 61: 146-149.

Dziuba, B., A. Babuchowski, M. Dziuba and D. Nałęcz, 2007. Identification of lactic acid bacteria using FTIR spectroscopy and artificial neural networks. Milchwissenschaft, 62: 28-32.

Huleihel, M., V. Pavlov and V. Erukhimovitch, 2009. The use of FTIR microscopy for the evaluation of anti-bacterial agents activity. J. Photochem. Photobiol. B: Biol., 96: 17-23. DOI: 10.1016/j.jphotobiol.2009.03.009
Igisu, M., Y. Ueno, M. Shimojima, S. Nakashima and S.M. Awramik et al., 2009. Micro-FTIR spectroscopic signatures of Bacterial lipids in Proterozoic microfossils. Precambrian Res., 173: 1926. DOI: 10.1016/j.precamres.2009.03.006

Johnsen, K. and P. Nielsen, 1999. Diversity of Pseudomonas strains isolated with King's B and Gould's S1 agar determined by repetitive extragenic palindromic-polymerase chain reaction, 16S rDNA sequencing and Fourier transform infrared spectroscopy characterisation. FEMS Microbiol. Lett., 173: 155-162. PMID: 10220892

Kirschner, C., K. Maquelin, P. Pina, N.A. Ngo-Thi and L.P. Choo-Smith et al., 2001. Classification and identification of enterococci: A comparative phenotypic, genotypic and vibrational spectroscopic study. J. Clin. Microbiol., 39: 1763-1770. DOI: 10.1128/JCM.39.5.1763-1770.2001

Mariey, L., J.P. Signolle, C. Amiel and J. Travert, 2001. Discrimination, classification, identification of microorganisms using FTIR spectroscopy and chemometrics. Vibrational Spectroscopy, 26: 151159. DOI: $10.1016 / \mathrm{S} 0924-2031(01) 00113-8$

Mossoba, M.M., S.F. Al-Khaldi, A. Jacobson, L.I. Segarra-Crowe and F.S. Fry, 2003. Application of a disposable transparent filtration membrane to the infrared spectroscopic discrimination among bacterial species. J. Microbiol. Methods, 55: 311 314. DOI: $10.1016 / \mathrm{S} 0167-7012(03) 00114-3$

Nauman, D., 2000. Infrared Spectroscopy in Microbiology. In: Encyclopedia of Analytical Chemistry, Meyers, R.A. (Ed.), John Wiley and Sons Australia, Limited, Chichester New York, ISBN-10: 9780471976707, pp: 102-131.

Naumann, D., D. Helm and H. Labischinski, 1991. Microbiological characterizations by FT-IR spectroscopy. Nature, 351: 81-82. DOI: 10.1038/351081a0

Rodriguez-Saona, L.E., F.M. Khambaty, F.S., Fry, E.M., Calvy, 2001. Rapid detection and identification of bacterial strains by Fourier transform near-infrared spectroscopy. J. Agric. Food Chem., 49: 574-579. DOI: $10.1021 / \mathrm{jf000776j}$

Sameli, J., A. Bleicher, C. Delbes-Paus, A. Kakouri and K. Neuhaus et al., 2011. FTIR-based polyphasic identification of lactic acid bacteria isolated from traditional Greek Graviera cheese. Food Microbiol., 28: 76-83. DOI: 10.1016/j.fm.2010.08.009 
Samelis, J., A. Kakouri, E. C. Pappa, B. B.Matijasic and M.D. Georgalaki et al., 2010. Microbial stability and safety of traditional Greek Graviera cheese: characterization of the lactic acid bacterial flora and culture-independent detection of bacteriocin genes in the ripened cheeses and their microbial consortia. J. Food Prot., 73: 1294-1303. PMID: 20615342

Timmins, E.M., S.A. Howell, B.K. Alsberg, W.C. Noble and R. Goodacre, 1998. Rapid differentiation of closely related Candida species and strains by pyrolysis-mass spectrometry and Fourier transforminfrared spectroscopy. J. Clin. Microbiol., 36: 367374. PMID: 9466743
Udelhoven, T., D. Nauman and J. Schmitt, 2000. Development of a hierarchical classification system with artificial neural networks and ft-ir spectra for the identification of bacteria. Applies Spectroscopy, 54: 1471-1479.

Weinrichter, B., W. Luginbuh, H. Rohm and J. Jimeno, 2001. Differentiation of facultatively heterofermentative Lactobacilli from plants, milk and hard type cheeses by SDS-PAGE, RAPD, FTIR, energy source utilisation and autolysis type. Lebens. Wiss. Technol., 34: 556-566. DOI: 10.1006/fstl.2001.0799 